\title{
A Study on Verification of the Dynamic Modeling for a Submerged Body Based on Numerical Simulation
}

\author{
Myungjun Jeon ${ }^{1}$, Tien Thua Nguyen ${ }^{1}$, Hyeon Kyu Yoon ${ }^{1, *}$, Hyeon Jin Cho ${ }^{2}$ \\ ${ }^{1}$ Department of Naval Architecture and Marine Engineering, Changwon National University, Korea \\ ${ }^{2}$ Agency of Defense Development, Korea \\ Received 29 May 2019; received in revised form 15 August 2019; accepted 18 November 2019 \\ DOI: https://doi.org/10.46604/ijeti.2020.4350
}

\begin{abstract}
This study proposed a procedure to identify maneuvering coefficients that brought about abnormal motions in the simulation of a submerged body. The first step in responding to abnormal motions was conducting stability analysis to determine whether the submerged body could be simulated. If doing so was feasible, sensitivity analysis was then performed to determine maneuvering coefficients that caused the abnormal motion in the simulation. Finally, we analyzed the order of maneuvering coefficients identified by the sensitivity analysis. We also compared it with empirical formulas and other results obtained from model tests. The dynamics model targeting a high-speed submerged body was indirectly verified by the above procedure. In this study, the effectiveness of the dynamic model was verified, and parameters causing the abnormal motion were identified in accordance with the developed procedure.
\end{abstract}

Keywords: 5-DOF equations of motion, maneuvering simulation, maneuvering coefficients, stability analysis, sensitivity analysis, abnormal motion

\section{Introduction}

The need for research and development of submerged bodies is increasing because of the increasing demand for exploring seabed and mineral resource and for strengthening national defense [1]. To design a submerged body with clearly defined mission and uses, system technology is required to merge various technologies such as hull design, propulsion, navigation, control, communication, energy, and sensor systems [2]. For hull design of a submerged body, in order for the submerged body to operate autonomously underwater, it should have proper maneuverability for its missions. Besides, various methods are required for estimating the maneuverability at the initial design stage [3]. In general, dynamic characteristics of a submerged body composed of a hull and a propeller; control planes are evaluated by estimating external forces based on Newton's second law. Prior to applying that law, external forces including hydrodynamic force, thrust, and control forces acting on each module are modeled; parameters constituting the model are estimated [4]. The simplest method for estimating these parameters is by using empirical formulas [5-6]. Although this method has the advantage of estimating maneuvering coefficients for the submerged body shape in a short time, its accuracy is not guaranteed. To estimate these parameters accurately, model tests such as captive model tests, propeller open-water tests are carried out in a model basin. Kim et al. [1] have performed a captive model test of a submerged body to obtain 6-DoF hydrodynamic coefficients and analyze the stability for vertical and horizontal planes. Park et al. [3] have performed a coning motion test and one of captive model tests. For a submerged body, Park et al. [3] also suggested a method to obtain roll hydrodynamic moment coefficients. Recently, CFD (Computational Fluid Dynamics)

* Corresponding author. E-mail address: hkyoon@ changwon.ac.kr

Tel.: +55-213-3683; Fax: +55-213-3689 
instead of model test has been used [7-10]. Nguyen et al. [7] have performed virtual captive model tests of a submerged body and presented its hydrodynamic damping coefficients. Sung and Park [9] have performed virtual captive model tests using CFD analysis to predict ship maneuverability and validated numerical methods by comparing its results with EFD (Experimental Fluid Dynamics) results. Another method for estimating these parameters is by using system identification method based on measured motion variables of a submerged body in operation [11]. Dynamic characteristics of a submerged body are generally evaluated by using such parameters obtained with various methods and numerical simulations [12-13]. Jeon et al. [13] have evaluated dynamic characteristics by performing numerical simulations and suggested guidelines for changes of design parameters based on dynamic characteristics. Dynamics models obtained from diverse methods not only can be used to predict dynamics characteristics or maneuvering performance, but also can be used to design controllers for motion control, path following, or performing its own missions [14-17]. Therefore, dynamics modeling for predicting accurate motions and obtaining stable dynamic system is important in the stage of initial concept design.

Not only maneuvering coefficients constituting external force models, but also the trend of its magnitude is diverse for a submerged body. When numerical simulations are used to evaluate dynamic characteristics, abnormal results are often encountered [18]. When more parameters constitute dynamic models, the process is more complex and time-consuming to find the cause of abnormal motions. This paper suggests a guideline for solving such problems that occur when numerical simulation gives abnormal results by applying Newton's second law after estimating maneuvering coefficients. When the simulation itself is impossible because of excessive motion and divergent phenomena, or when results are very different from those of existing similar submerged bodies, we introduce a method to identify parameters that cause the abnormal symptom among various parameters of the dynamics model. The proposed method in this paper can be used to find causes of abnormal results that occur during numerical simulations. It can also be used to re-validate abnormal parameters.

\section{Mathematical Model for a Submerged Body}

\subsection{Coordinate systems}

Fig. 1 shows right-hand orthogonal coordinate systems consisting of an earth-fixed coordinate system $\left(O-x_{o} y_{o}, O-x_{o} z_{o}\right)$ and a body-fixed coordinate system $(o-x y, o-x z)$ for horizontal and vertical planes, respectively. The trajectory of a submerged body is defined in the earth-fixed coordinate system. The hydrodynamic force acting on a submerged body is defined in the body-fixed coordinate system.

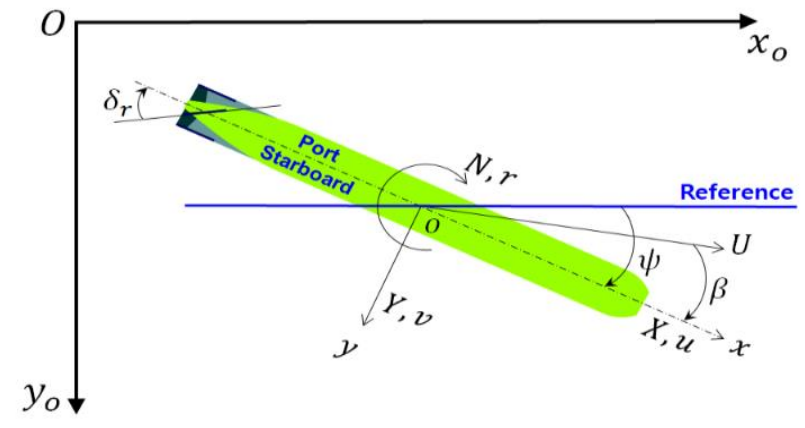

(a) Horizontal plane $\left(O-x_{o} y_{o}\right)$

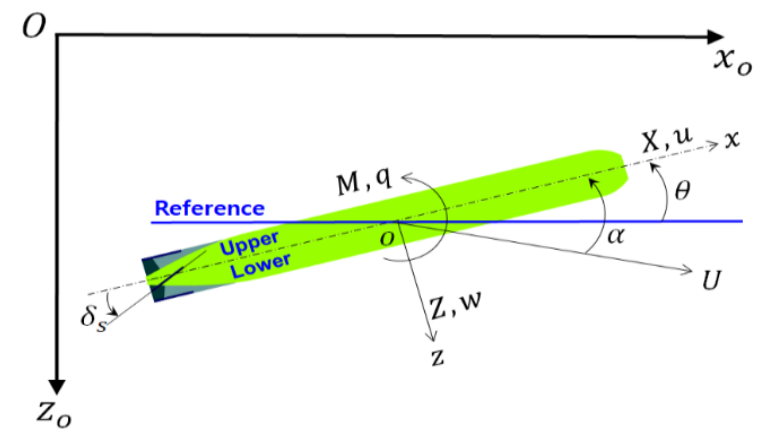

(b) Vertical plane $\left(O-x_{o} z_{o}\right)$

Fig. 1 Description of coordinate systems

\subsection{5-DoF equations of motions}

Assume that the coupled effect between horizontal and vertical planes is small because of symmetry of $o-x y$ and $o-x z$ plane, and that rolling is prevented by controlling the stern planes. The decoupled equations of motion for three horizontal motions and two vertical motions are defined as Eqs. (1)-(5) respectively. 


$$
\begin{aligned}
& m(\dot{u}-v r)=X_{H D}+X_{H S}+H_{C}+X_{T} \\
& m(\dot{v}+u r)=Y_{H D}+Y_{H S}+Y_{C}+Y_{T} \\
& I_{z z} \dot{r}=N_{H D}+N_{H S}+N_{C}+N_{T} \\
& m\left(\dot{w}-u q-z_{G} q^{2}\right)=Z_{H D}+Z_{H S}+Z_{C}+Z_{T} \\
& I_{y y} \dot{q}+m\left(z_{G} w q\right)=M_{H D}+M_{H S}+M_{C}+M_{T}
\end{aligned}
$$

where $m, I_{y y}$, and $I_{z z}$ are mass and mass moment of inertia in the y-axis and z-axis, respectively. Parameters defined on the right side of Eqs. (1)-(5) represent external forces acting on the submerged body. The subscript HD expressed as Eqs. (6)-(10) means hydrodynamic forces caused by its motions such as velocity and acceleration. HS expressed as Eqs. (11)-(15) means hydrostatic forces caused by by its gravitational force, buoyant force, and attitude. $C$ and $T$ expressed as Eqs. (16)-(25) mean control forces caused by rudder, stern planes deflections, and thrust. The kinematic relationship between the motion in body-fixed and earth-fixed coordinate system is defined as Eqs. (26)-(27) using Euler 3-2-1 transformation.

$$
\begin{aligned}
& X_{H D}=X_{\dot{u}} \dot{u}+X_{u|u|} u|u|+X_{v v} v^{2}+X_{v r} v r+X_{w q} w q+X_{q q} q^{2}+X_{r r} r^{2} \\
& Y_{H D}=Y_{\dot{v}} \dot{v}+Y_{\dot{r}} \dot{r}+Y_{v} v+Y_{v|v|} v|v|+Y_{r} r+Y_{r|r|} r|r| \\
& N_{H D}=N_{\dot{v}} \dot{v}+N_{\dot{r}} \dot{r}+N_{v} v+N_{v|v|} v|v|+N_{r} r+N_{r|r|} r|r| \\
& Z_{H D}=Z_{\dot{w}} \dot{w}+Z_{\dot{q}} \dot{q}+Z_{w} w+Z_{w|w|} w|w|+Z_{q} q+Z_{q|q|} q|q| \\
& M_{H D}=M_{\dot{w}} \dot{w}+M_{\dot{q}} \dot{q}+M_{w} w+M_{w|w|} w|w|+M_{q} q+M_{q|q|} q|q| \\
& X_{H S}=-(m g-p g \nabla) \sin \theta \\
& Y_{H S}=0 \\
& N_{H S}=0 \\
& Z_{H S}=(m g-p g \nabla) \cos \theta \\
& M_{H S}=-\left(z_{G} m g-z_{B} p g \nabla\right) \sin \theta \\
& X_{C}=0 \\
& Y_{C}=Y_{\delta_{r}} \delta_{r} \\
& N_{C}=N_{\delta_{r}} \delta_{r} \\
& Z_{C}=Z_{\delta_{s}} \delta_{s} \\
& M_{C}=M_{\delta_{s}} \delta_{s} \\
& X_{T}=-X_{u|u|} U_{C}\left|U_{C}\right| \\
& Y_{T}=0 \\
& N_{T}=0 \\
& Z_{T}=0 \\
& M_{T}=0 \\
& =0
\end{aligned}
$$




$$
\left[\begin{array}{l}
\dot{x}_{o} \\
\dot{y}_{o} \\
\dot{z}_{o} \\
\dot{\theta} \\
\dot{\psi}
\end{array}\right]=C_{b}^{n}\left[\begin{array}{c}
u \\
v \\
w \\
q \\
r
\end{array}\right]
$$

where

$$
C_{b}^{n}=\left[\begin{array}{ccccc}
\cos \psi \cos \theta & -\sin \psi & \cos \psi \sin \theta & 0 & 0 \\
\sin \psi \cos \theta & \cos \psi \cos \theta & \sin \psi \sin \theta & 0 & 0 \\
-\sin \theta & 0 & \cos \theta & 0 & 0 \\
0 & 0 & 0 & 1 & 0 \\
0 & 0 & 0 & 0 & 1 / \cos \theta
\end{array}\right]
$$

where $u, v, w, q, r, \delta_{r}, \delta_{s}$, and $U_{c}$ are the axial, lateral velocity, vertical velocity, pitch and yaw rate, rudder angle, stern plane angle, and command speed, respectively. The point marked at the top of motion variables means the time rate of change. Parameters described in Eqs. (6)-(10) and Eqs. (16)-(20) called maneuvering coefficients are partial derivatives of subscript motion variables. The thrust $X_{T}$ is acting in the direction opposite to the resistance. It is simply modeled without propeller characteristics. To obtain added mass coefficients $Z_{w}, Z_{q}, M_{w}$, and $M_{q}$, dynamic tests such as pure heave and pure pitch tests should be performed. Therefore, maneuvering coefficients were obtained by performing virtual VPMM (Vertical Planar Motion Mechanism) tests using unsteady CFD analysis in this study. The purpose of this study was to find parameters that caused abnormal motion during numerical simulation. Thus, estimating coefficients using CFD was omitted in this paper. The non-dimensionalization of force, moment, mass, and mass moment of inertia are expressed as:

$$
\begin{aligned}
& \text { Force }^{\prime}=\frac{\text { Force }}{0.5 \rho A U^{2}} \\
& \text { Moment }{ }^{\prime}=\frac{\text { Moment }}{0.5 \rho A L U^{2}} \\
& m^{\prime}=\frac{m}{0.5 \rho A L} \\
& I_{y y}{ }^{\prime}=\frac{I_{y y}}{0.5 \rho A L^{3}} \\
& I_{z z}{ }^{\prime}=\frac{I_{z z}}{0.5 \rho A L^{3}}
\end{aligned}
$$

where $\rho, A, L$, and $U$ mean water density, cross-section area in the middle of xy-plane, body length, and speed, respectively.

\section{Parameter Identification based on the Numerical Simulation}

\subsection{A submerged body}

The target submerged body is symmetric for the $y$-axis and the z-axis. Principal particulars are listed in Table 1. The origin of the body-fixed coordinate system is located in the center of buoyancy. Products of inertia $I_{y z}$ and $I_{z x}$ are assumed to be zero because the shape of the body is symmetric with respect to the two planes. Additionally, the vertical center of gravity is 
located sufficiently below the vertical center of buoyancy to ensure static stability. Also, the target submerged body is operating at a high speed with the assumption of small drift angle $\beta$ and angle of attack $\alpha$. Upper and lower rudders have symmetrical shapes. The right and left stern planes also have symmetrical shapes. Upper rudder, lower rudder, the right stern plane, and the left stern plane rotate simultaneously.

Table 1 Principal particulars of the submerged body

\begin{tabular}{|c|c|}
\hline Item [unit] & Value \\
\hline Length $[m]$ & 1.94 \\
\hline Diameter $[m]$ & 0.21 \\
\hline Wetted Surface Area $\left[\mathrm{m}^{2}\right]$ & 1.19 \\
\hline Block coefficient, $C_{b}[-]$ & 0.656 \\
\hline Prismatic coefficient, $C_{p}[-]$ & 0.835 \\
\hline Weight $[N]$ & 1049.7 \\
\hline Buoyancy $[N]$ & 589.9 \\
\hline Center of gravity $\left[\begin{array}{lll}x_{G} & y_{G} & z_{G}\end{array}\right][m]$ & {$\left[\begin{array}{lll}0.00 & 0.00 & 0.03\end{array}\right]$} \\
\hline Center of buoyancy $\left[\begin{array}{lll}x_{B} & y_{B} & z_{B}\end{array}\right][m]$ & {$\left[\begin{array}{lll}0.00 & 0.00 & 0.00\end{array}\right]$} \\
\hline Mass moment of inertia $\left[\begin{array}{ll}I_{y y} & I_{z z}\end{array}\right]\left[\mathrm{kg} \cdot \mathrm{m}^{2}\right]$ & {$\left[\begin{array}{ll}31.6 & 31.6\end{array}\right]$} \\
\hline Rudder position from the $\mathrm{X}$-origin $[m]$ & -0.86 \\
\hline Stern plane position from the $\mathrm{x}$-origin [m] & -0.86 \\
\hline Rudder area $\left[\mathrm{m}^{2}\right]$ & $2.07 \mathrm{E}-2$ \\
\hline Stern plane area $\left[\mathrm{m}^{2}\right]$ & $2.07 \mathrm{E}-2$ \\
\hline
\end{tabular}

\subsection{Abnormal motion occurrence}

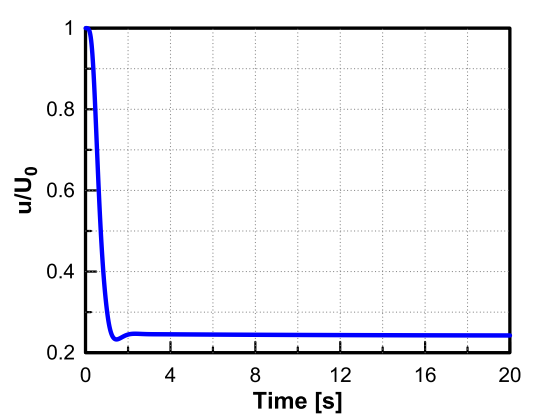

(a) Non-dimensional axial velocity

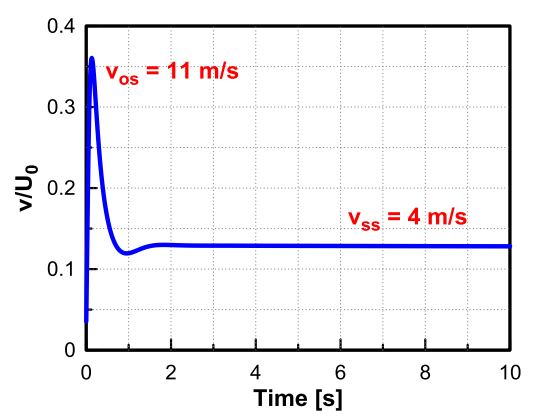

(b) Non-dimensional lateral velocity

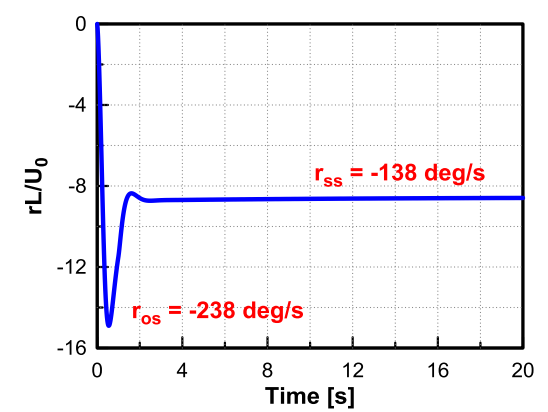

(c) Non-dimensional yaw rate

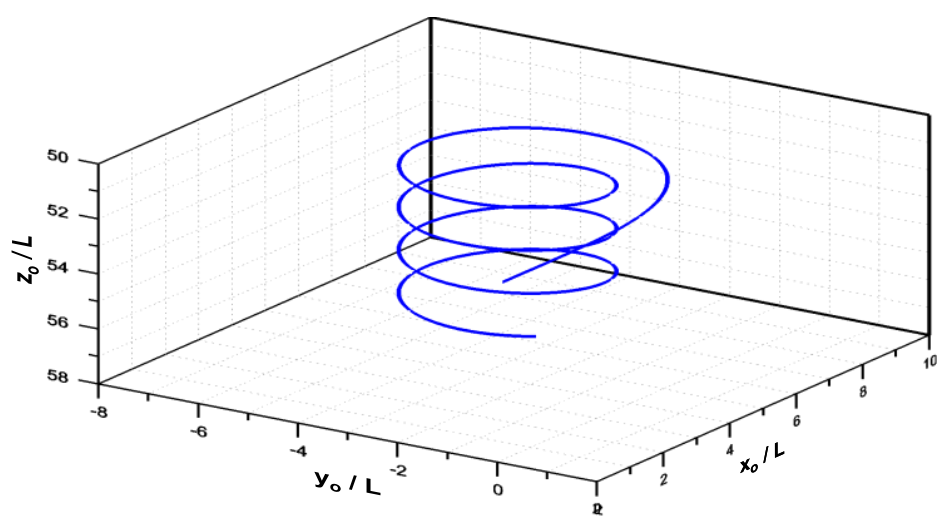

(d) Non-dimensional trajectory (initial $Z_{0}=100 \mathrm{~m}$ )

Fig. 2 Turning simulation results of the target submerged body $\left(\delta_{r}=-30^{\circ}, \delta_{r}=0^{\circ}\right)$

By using equations of motion written in Eqs. (1)-(5), numerical simulation was performed to predict dynamic characteristics. Fig. 2 shows turning simulation results of the submerged body when it changes -35 degrees of the rudder angle. $U_{0}$ on the y-axis in Fig. 2(a)-2(c) means the initial speed expressed as $\sqrt{u_{0}^{2}+v_{0}^{2}+w_{0}^{2}}$ at which a submerged body goes straight 
before deflecting the rudder. As a dimensional value, the overshoot of lateral velocity $v_{o s}$ and yaw rate $r_{o s}$ are abnormally large at $7.5 \mathrm{~m} / \mathrm{s}$ and $-238 \% \mathrm{~s}$, respectively. Also, the dimensional steady yaw rate $r_{s s}$ is abnormally fast, $-138 \%$. As shown in Fig. 2(d), since the submerged body has a negative buoyancy, the depth drops from the initial depth 100 meters when it turns. This phenomenon is caused by the hydrostatic force $Z_{H S}$ and $M_{H S}$ described in Eqs. (11)-(15) if there is no coupled effect of hydrodynamic forces between the horizontal plane and the vertical plane.

\subsection{The procedure to identify model parameters causing abnormal motion}

It is difficult to identify parameters that cause the abnormal motion when carrying out the numerical simulation since there are many parameters consisting of external forces. In this paper, we defined three kinds of abnormal motion that could occur in maneuvering simulations. As shown in Table 2 and Fig. 3, the procedure for identifying such parameters has a great effect on abnormal motions mentioned in Table 2.

Table 2 Types of abnormal motions

\begin{tabular}{cr}
\hline Case & Description \\
\hline Case 1 & The maneuvering simulation is impossible. \\
Case 2 & The overshoot of the lateral velocity or yaw rate is abnormally large when it turns. \\
Case 3 & The yaw rate in steady state is abnormally large or small when it turns \\
\hline
\end{tabular}

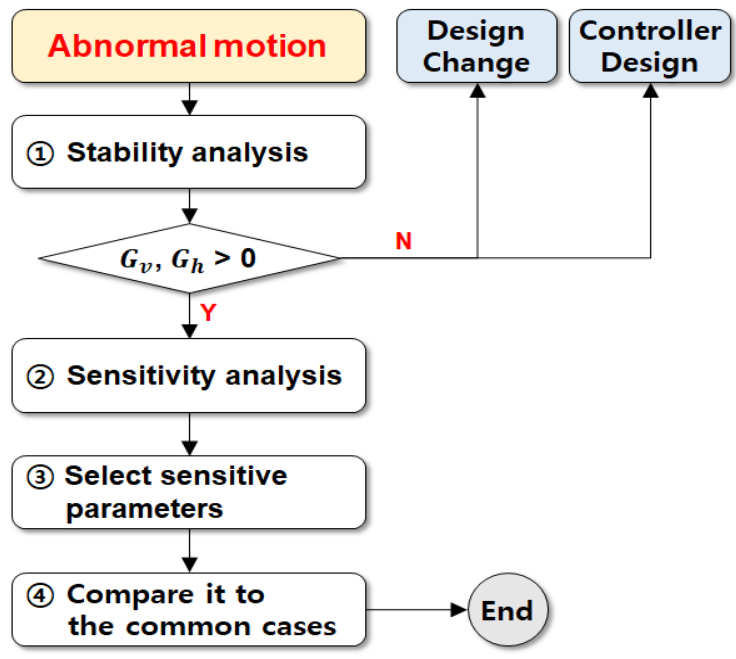

Fig. 3 Procedure for identifying parameters mainly affecting abnormal motions.

\subsection{Stability analysis}

For Case 1 described in Table 2, the first step is to evaluate the dynamic stability of the system to find out whether it can be simulated. The dynamic stability can be divided into two kinds. One is the dynamic stability for roll motion. To be statically stable, BG $\left(=z_{G}-z_{B}\right)$ should be positive [19]. Roll hydrodynamic damping moment also has to be sufficiently large to be dynamically stable. If a submerged body does not have static or dynamic stability, then numerical simulation is impossible since the roll motion diverges to infinity. Commonly, in case of a high-speed submerged body, roll hydrodynamic damping moment is forcibly generated by stern planes. Therefore, we assumed that roll was stable and applied 5-DoF equations of motion except for roll motion. The other is dynamic stability of vertical and horizontal motion. This means that the system can return to the steady state after the initial disturbance. If the system does not ensure dynamic stability, certain motion variables will diverge as time goes on. In this case, it is possible to simulate by applying controllers to prevent divergence of vertical or horizontal motion variables. However, prior to designing the controller, it is recommended to change design parameters to 
ensure dynamic stability. For the submerged body free of horizontal and vertical motions, stability analysis should be done by checking the sign of real parts of eigenvalues in perturbed state equations [4-5] or by calculating the stability margin in such a way that the dynamic stability is ensured when static stability is secured [4]. In this study, we evaluated the dynamic stability by using the stability margin. Horizontal and vertical plane stability indices can be obtained from Eq. (33) and Eq. (34) respectively. To ensure dynamic stability, stability indices should be positive. For target submerged body, dynamic stability is ensured because both stability indices are equal to 0.6. Therefore, target submerged body is a system capable of numerical simulation.

$$
\begin{gathered}
G_{h}=1-\frac{N_{v}^{\prime}\left(Y_{r}^{\prime}-m^{\prime}\right)}{Y_{v}^{\prime}\left(N_{v}^{\prime}-m^{\prime} x_{G}^{\prime}\right)} \\
G_{v}=1-\frac{M_{v}^{\prime}\left(Z_{q}^{\prime}+m^{\prime}\right)}{Z_{w}^{\prime}\left(M_{q}^{\prime}-m^{\prime} x_{G}^{\prime}\right)}
\end{gathered}
$$

\subsection{Sensitivity analysis}

If numerical simulation is feasible because dynamic stability is ensured, the next step is to perform a sensitivity analysis to determine maneuvering coefficients that contribute to current dynamic characteristics of the target submerged body. Sensitivity analysis for the maneuverability has been studied variously [20-23]. Sensitivity is one of dynamic characteristics with respect to maneuvering coefficients as suggested by Jeon et al. [13]. Sensitivity analysis for the turning ability corresponding to Cases 2 and 3 in Table 2 can be done by using a direct method if hydrodynamic coefficients constituting the right side of Eqs. (1)-(3) are composed of the only linear coefficients [13]. However, since the present system includes nonlinear coefficients, it is necessary to calculate the sensitivity using an indirect method that carries out numerical simulation each time by adjusting specific maneuvering coefficients. In this study, the overshoot of sway velocity and yaw rate in steady state are selected as abnormal motions based on Cases 2 and 3. Their definitions are shown in Fig. 3. The sensitivity of abnormal motions for each maneuvering coefficient can be expressed by Eq. (35). It is normalized as Eq. (36). Vectors $\underline{R}_{0}$ and $\underline{H}_{0}$ constituting Eq. (36) represents abnormal motion indices and maneuvering coefficients before adjustment respectively. Vector $\underline{R}$ in Eq. (37) means abnormal motion indices after adjusting maneuvering coefficients. Vector $\underline{H}$ in Eq. (38) is a vector consisting of maneuvering coefficients after adjustment. Tha matrix in Eq. (39) is a Jacobian matrix which is expressed as a partial derivative of abnormal motion indices vector with respect to maneuvering coefficients vector. The sensitivity matrix in Eq. (39) is valid when vector $\underline{H}$ varies in a small range. In this study, components of sensitivty matrix are calculated by changing vector $\underline{H}$ by $5 \%$.

$$
\begin{aligned}
& \frac{\partial \underline{R}}{\partial \underline{H}} \approx \frac{\underline{R}(\underline{H}+\Delta \underline{H})-\underline{R}(\underline{H})}{\Delta \underline{H}} \\
& S_{H}^{R}=\left\{\operatorname{diag}\left(\left|\underline{R}_{0}\right|\right)\right\}^{-1}\left(\frac{\partial \underline{R}}{\partial \underline{H}}\right) \operatorname{diag}\left(\left|\underline{H}_{0}\right|\right)
\end{aligned}
$$

where

$$
\underline{R}=\left[\begin{array}{lll}
v_{o s} & r_{o s} & r_{s s}
\end{array}\right]^{T}
$$




$$
\begin{aligned}
& \underline{H}=\left[\begin{array}{lllllll}
Y_{\dot{v}}^{\prime} & Y_{\dot{r}}^{\prime} & Y_{v}^{\prime} & Y_{v|v|}^{\prime} & Y_{r}^{\prime} & \cdots & N_{\delta_{r}}{ }^{\prime}
\end{array}\right]^{T} \\
& \frac{\partial \underline{R}}{\partial \underline{H}}=\left[\begin{array}{ccc}
\frac{\partial v_{\mathrm{os}}}{\partial Y_{\dot{v}}^{\prime}} & \frac{\partial v_{\mathrm{os}}}{\partial Y_{\dot{v}}^{\prime}} & \frac{\partial v_{\mathrm{os}}}{\partial Y_{\dot{v}}^{\prime}} \\
\frac{\partial v_{\mathrm{os}}}{\partial Y_{v}^{\prime}} & \frac{\partial v_{\mathrm{os}}}{\partial Y_{v}^{\prime}} & \frac{\partial v_{\mathrm{ss}}}{\partial Y_{v}^{\prime}} \\
\vdots & \vdots & \vdots \\
\frac{\partial v_{\mathrm{os}}}{\partial N_{\delta_{r}}^{\prime}} & \frac{\partial v_{\mathrm{os}}}{\partial N_{\delta_{r}}^{\prime}} & \frac{\partial v_{\mathrm{ss}}}{\partial N_{\delta_{r}}{ }^{\prime}}
\end{array}\right]
\end{aligned}
$$

Fig. 4 shows an example of time series of abnormal motions described in Table 2. $v_{o s}$ shown in Fig. 4(a) means overshoot of lateral velocity in time series. $r_{o s}$ and $r_{s s}$ shown in Fig. 4(b) mean overshoot of yaw rate and the steady in steady state, respectively.

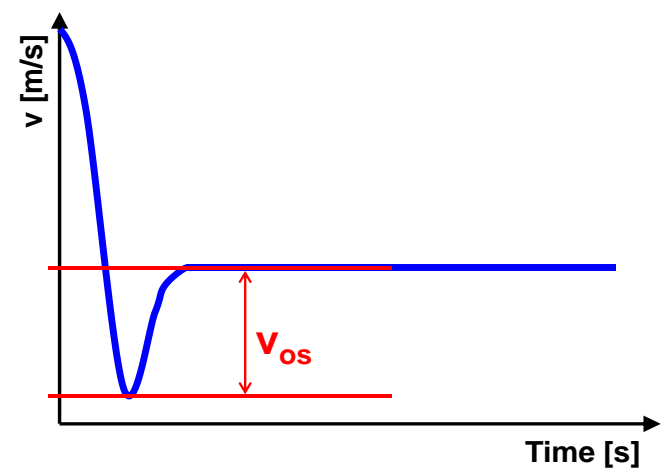

(a) $v_{o s}$

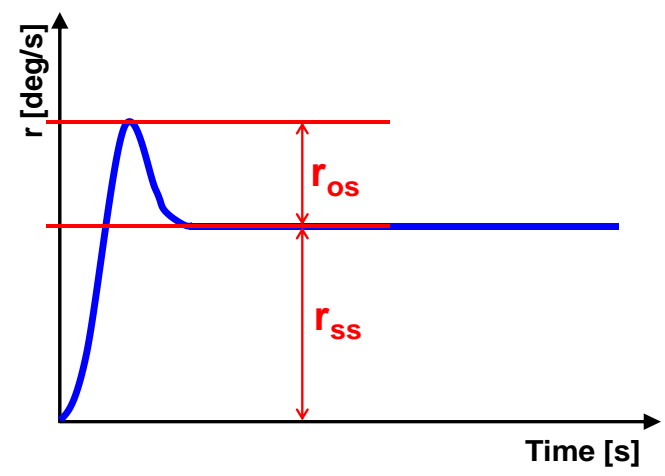

(b) $r_{o s}$ and $r_{s s}$

Fig. 4 The definition of $v_{o s}, r_{o s}$, and $r_{s s}$ in time series

\subsection{Sensitive parameter identification}

The normalized sensitivity matrix $S_{H}^{R}$ mentioned in Section 3.5 is described in Table 3. The sign in parentheses is the correlation between abnormal motion and maneuvering coefficients. In Table 3, relatively sensitive maneuvering coefficients are shaded. By performing procedures described in Sections 3.4 and 3.5, maneuvering coefficients required to be revalidated are reduced to 7 out of a total of 14 coefficients. Sensitive parameter identification can be summarized as follows.

1. A large order of sensitivity means that it has a great influence on the state of motion of the submerged body regardless of whether the value of maneuvering coefficients is correct or not.

2. Overall, it is sensitive to linear stability coefficients, $Y_{v}^{\prime}, Y_{r}^{\prime}, N_{v}{ }^{\prime}, N_{r}{ }^{\prime}$ and control plane coefficients, $Y_{\delta_{r}}{ }^{\prime}, N_{\delta_{r}}{ }^{\prime}$. These coefficients have a relatively large order in terms of sensitivity.

3. In the initial transient section, overshoots $v_{o s}$ and $r_{o s}$ are sensitive to the $Y$-force related coefficients. It can be indirectly assumed that overshoot $r_{o s}$ is caused by coupled effects with the sway velocity overshoot $v_{o s}$.

4. The yaw rate in steady state $r_{s s}$ is sensitive to coefficients associated with the $N$-moment, $N_{v}{ }^{\prime}, N_{r}{ }^{\prime}, N_{\delta_{r}}{ }^{\prime}$.

5. In conclusion, maneuvering coefficients needed to be revalidated are as follows: $Y_{\dot{r}}^{\prime}, Y_{v}^{\prime}, Y_{r}^{\prime}, Y_{\delta_{r}}^{\prime}, N_{v}^{\prime}, N_{r}^{\prime}$, and $N_{\delta_{r}}{ }^{\prime}$. 
Table 3 Normalized sensitivity matrix $S_{H}^{R}$

\begin{tabular}{|c|c|c|c|}
\hline Sensitivity of & $v_{o s}$ & $r_{o s}$ & $r_{s s}$ \\
\hline$Y_{\dot{v}}^{\prime}$ & $0.039(-)$ & $0.023(+)$ & $0.000(+)$ \\
\hline$Y_{\dot{r}}^{\prime}$ & $0.206(+)$ & $0.221(-)$ & $0.001(-)$ \\
\hline$Y_{v}^{\prime}$ & $0.175(-)$ & $0.218(+)$ & $0.120(+)$ \\
\hline$Y_{r}^{\prime}$ & $0.106(-)$ & $0.116(+)$ & $0.058(+)$ \\
\hline$Y_{v|v|}^{\prime}$ & $0.053(-)$ & $0.077(+)$ & $0.049(+)$ \\
\hline$Y_{r|r|}^{\prime}$ & $0.010(+)$ & $0.012(-)$ & $0.007(-)$ \\
\hline$Y_{\delta_{r}}^{\prime}$ & $0.128(+)$ & $0.141(-)$ & $0.064(-)$ \\
\hline$N_{\dot{v}}^{\prime}$ & $0.002(-)$ & $0.006(+)$ & $0.000(+)$ \\
\hline$N_{\dot{r}}^{\prime}$ & $0.001(-)$ & $0.004(+)$ & $0.001(-)$ \\
\hline$N_{v}^{\prime}$ & $0.320(+)$ & $0.294(-)$ & $0.349(-)$ \\
\hline$N_{r}^{\prime}$ & $0.456(-)$ & $0.497(+)$ & $0.503(+)$ \\
\hline$N_{v|v|}^{\prime}$ & $0.092(-)$ & $0.059(+)$ & $0.116(-)$ \\
\hline$N_{r|r|}^{\prime}$ & $0.009(+)$ & $0.010(-)$ & $0.009(+)$ \\
\hline$N_{\delta_{r}}^{\prime}$ & $0.272(-)$ & $0.324(+)$ & $0.265(+)$ \\
\hline & &
\end{tabular}

\subsection{Comparison with the common cases}

First, seven sensitive coefficients that need to be revalidated should be confirmed by checking the order of the value. The best approach is to compare maneuvering coefficients obtained by captive model tests with a similar type of model. If there are no model test results, it is a good approach to compare the coefficient with the one calculated from the empirical formula when the body is replaced with equivalent wings [5]. For the target submerged body in this study, there are results from captive model tests for a submerged body with a similar shape operated for similar purposes. The shape and principal particulars of the pre-existing submerged body are similar to the target submerged body, and the operating purpose is the same as the target one.

Fig. 5 compares absolute values representing relative differences between the target and pre-existing submerged body. Compared with coefficients of the pre-existing submerged body, control plane coefficients $Y_{\delta_{r}}{ }^{\prime}$ and $N_{\delta_{r}}{ }^{\prime}$ seem to have large differences. This confirms that the order of values is different.

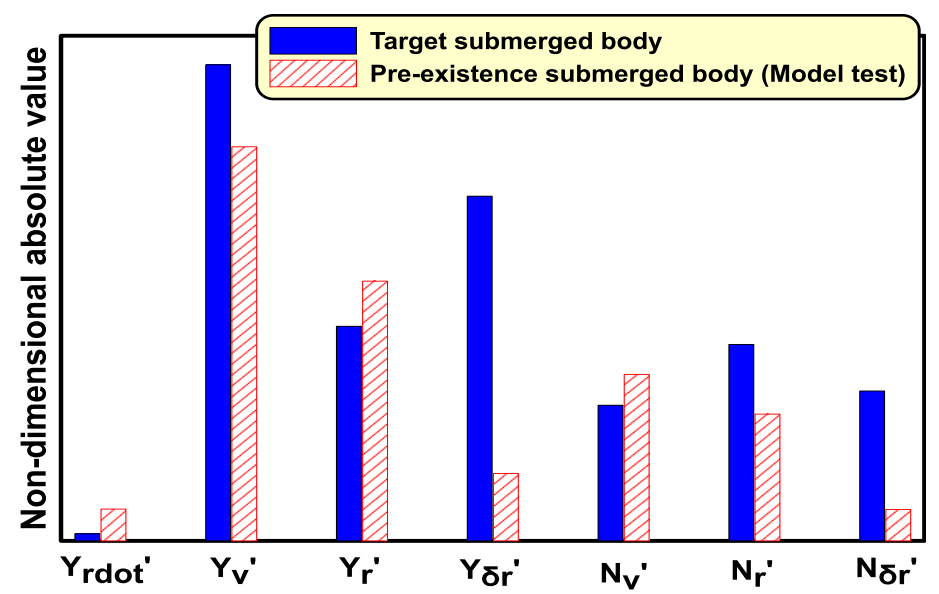

Fig. 5 Comparison of the maneuvering coefficients between the target and pre-existence submerged body 
Abnormal motions shown in Fig. 2 arise because $N_{r}{ }^{\prime} / N_{\delta_{r}}{ }^{\prime}$ and $Y_{v}{ }^{\prime} / Y_{\delta_{r}}{ }^{\prime}$ are small and more precise while absolute values of control plane coefficients are abnormally large. In this case, it is necessary to confirm the ratio of the hull-side projected area and the rudder area. Hydrodynamic damping coefficients $Y_{v}^{\prime}$ and $N_{r}^{\prime}$ indicate force and moment acting on a hull when the hull motion occurs. On the other hand, $Y_{\delta_{r}}{ }^{\prime}$ and $N_{\delta_{r}}{ }^{\prime}$ represent force and moment acting on the hull when the rudder is deflected. Commonly, the order of control coefficients $Y_{\delta_{r}}{ }^{\prime}$ and $N_{\delta_{r}}{ }^{\prime}$ is smaller than the order of hull damping coefficient $Y_{v}{ }^{\prime}$ and $N_{v}^{\prime}$. However, in this study, the relationship between hull design parameters and maneuvering coefficients of submerged bodies is not explained in detail. Whether values are right or wrong is not the point. The point is that these coefficients are main causes of abnormal motions as shown in Fig. 2.

\section{Modification of Abnormal Parameters}

\subsection{Recalculation using CFD analysis}

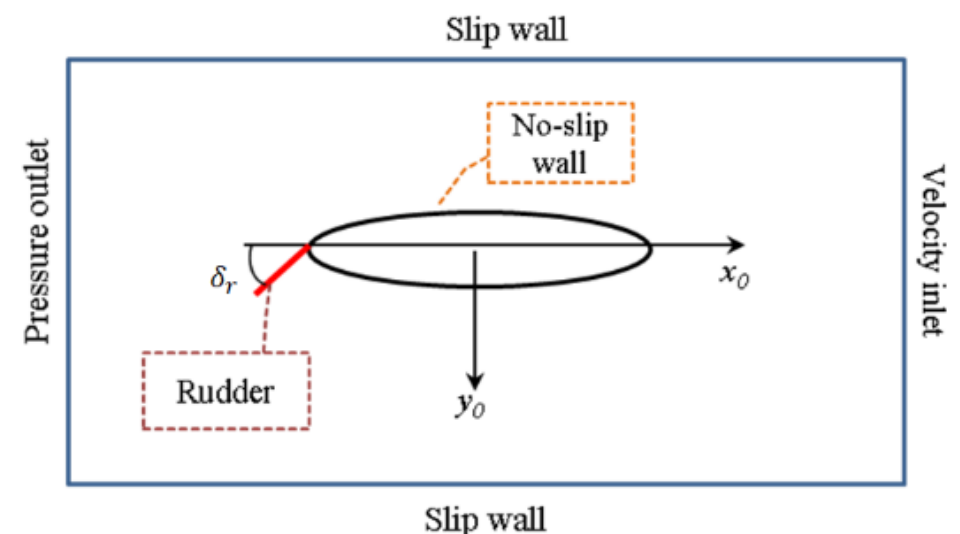

Fig. 6 Analysis domain for virtual static rudder tests

Table 4 Brief summary for CFD analysis

\begin{tabular}{|c|c|}
\hline Item & Numerical method \\
\hline Turbulence model & Realizable k- $\varepsilon$ \\
\hline Algorithm & Semi-Implicit Method for Pressure-Linked Equations \\
\hline Interpolation & Least-Squares Cell-based \\
\hline Interpolation method for pressure & Standard \\
\hline Spatial discretization scheme & Second Order Upwind \\
\hline Number of cells & $6,800,000$ elements \\
\hline
\end{tabular}

Main parameters identified in the previous section are control plane coefficients that can be estimated by performing static rudder tests. Therefore, virtual static rudder tests using CFD analysis are redone to revalidate. By considering that the fluid domain has great significance before starting the simulation, a rectangular shape is selected to represent the fluid domain covering the AUV. Dimensions of the domain are $5 \mathrm{~L}$ in length, $4 \mathrm{~L}$ in width, and $3 \mathrm{~L}$ in depth. In addition, physical conditions are applied to boundaries of the domain. The front face and back face are assigned to be velocity inlet and pressure outlet, respectively. The top face and bottom face of the domain are considered as symmetric conditions. Slip condition is set for sidewalls while a no-slip condition is specified for the AUV. Fig. 6 illustrates the fluid domain and boundary conditions for the virtual static rudder test. The software for CFD analysis is ANSYS FLUENT version 19.2. The analytical methods and generation of grids in the position of the tail cone are shown in Table 4 and Fig. 7, respectively. For CFD-based analysis, the solution is obtained when the residual decreases to 10-5. Static rudder tests are performed to obtain the force acting on the hull by changing rudder angles with zero drift angle. The force caused by the rudder deflection is calculated at $5^{\circ}$ intervals from $0^{\circ}$ to the maximum rudder angle. $Y_{\delta_{r}}$ and $N_{\delta_{r}}$ are partial derivatives of the Y-force and N-moment with respect to rudder angles. 
Fig. 8 shows the results of virtual static rudder tests comparing results of former calculation with the recalculation. In comparison with former results, present results showed a small slope at the point of zero degrees, which implied that that there was a problem in the former calculation process and that the identification procedure for finding abnormal parameters was valid.

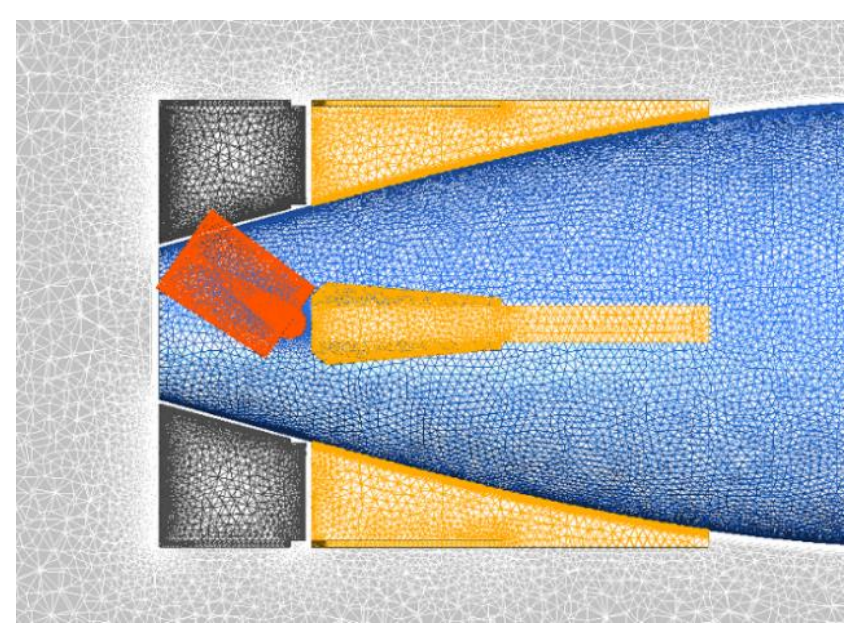

Fig. 7 Grids for CFD analysis (tailcone view, xy-plane)

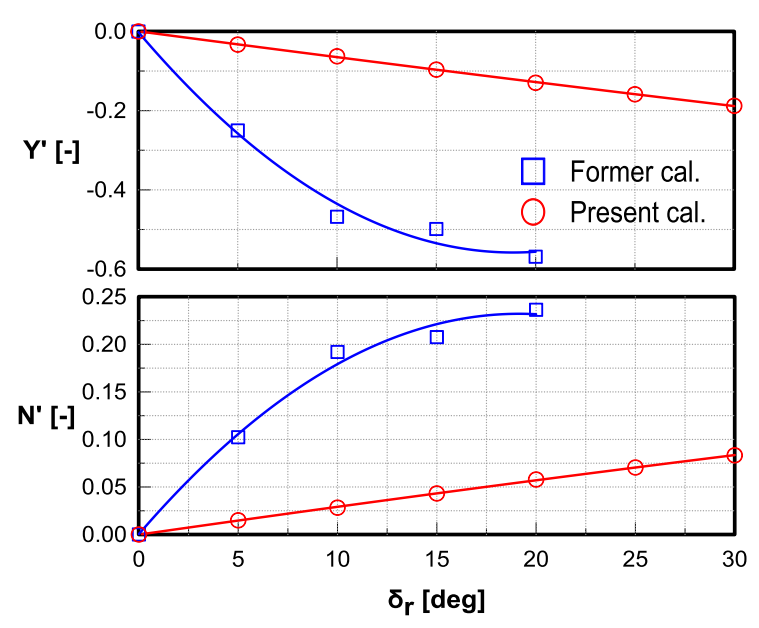

Fig. 8 The results of static rudder tests using CFD

\subsection{Dynamic characteristics after modifications}

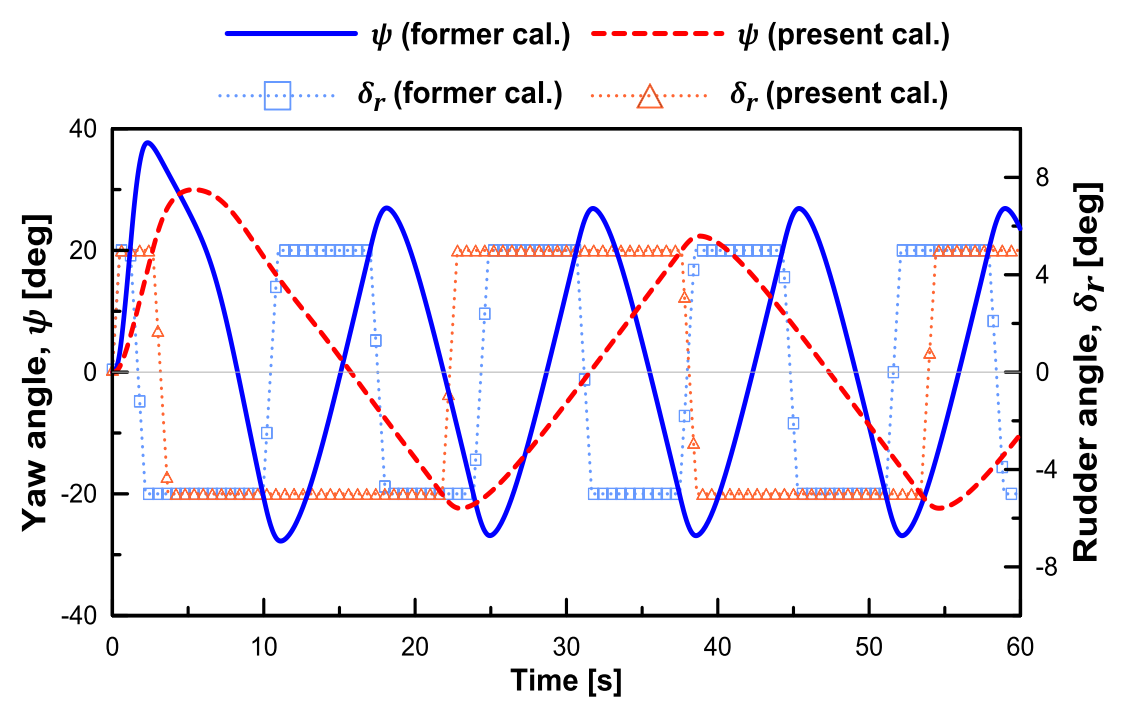

Fig. 10 5-20 horizontal zig zag simulation results of the target submerged body using formal and present control plane coefficients

Results of turning simulations using modified control-plane coefficients with a constant speed are shown in Fig. 9. Results of comparison between former and present calculations are also shown in Fig. 9. It can be seen that as control-plane coefficients become smaller, and overshoots of the sway velocity and yaw rate decrease to a common level. Fig. 10 shows comparison of 5-20 horizontal zig zag simulations using formal and present control plane coefficients. In the formal results denoting blue solid lines, the response of yaw with respect to rudder angles is too fast due to large control plane coefficients. Fig. 11 shows results of meander maneuver simulations. Meander maneuver was performed to investigate if the submerged body returned to a stable state after a disturbance of the pitch angle [24]. In the meander simulation, when the speed reaches the command speed $U_{c}=20$ knots, the stern plane is commanded a certain angle $\delta_{s}=10^{\circ}$. If the vertical stability is ensured, the pitch angle returns to or close to $0^{\circ}$. Additionally, the depth is eventually maintained. If the gravitational force is larger than buoyant force, vertical velocity occurs as shown in Fig. 11(a). Pitch angle is also changed due to the coupled effect between 
heave and pitch like $M_{w} w$ and $M_{w|w|} w|w|$. As a result, the depth changes not only the meander maneuver, but also straight motion as shown in Fig. 11(b) expressed as circle symbols. In this case, the control force should be generated to cancel constant vertical motions. If pitch angle is positive as shown in Fig. 11(b), the diving depth should decrease. The decrease of diving depth with its eventual increases is due to the effect of negative buoyancy.

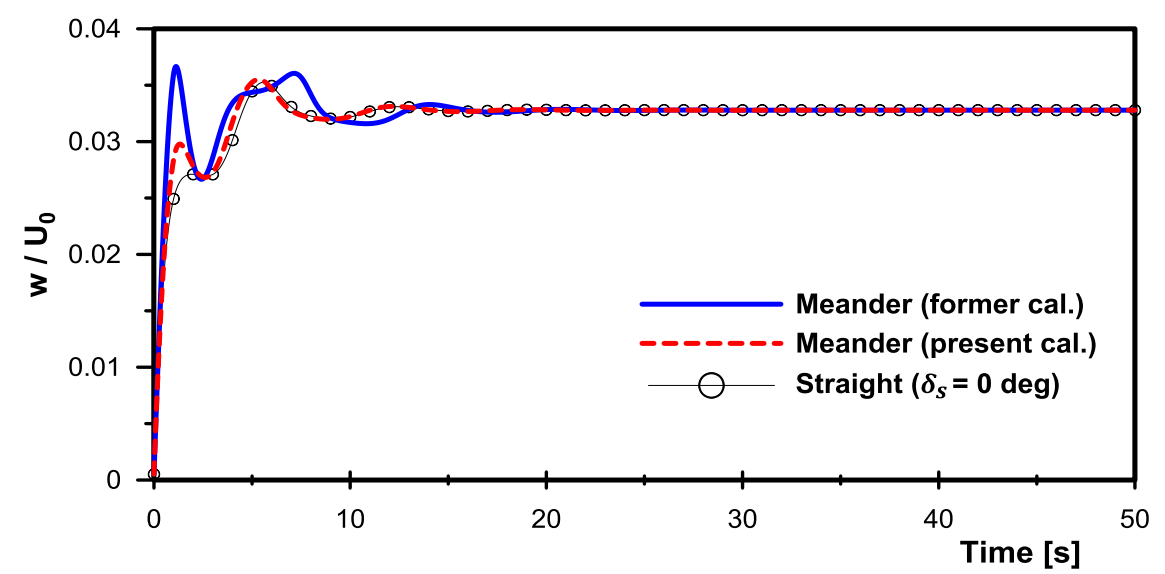

(a) Time series of non-dimensional vertical velocity

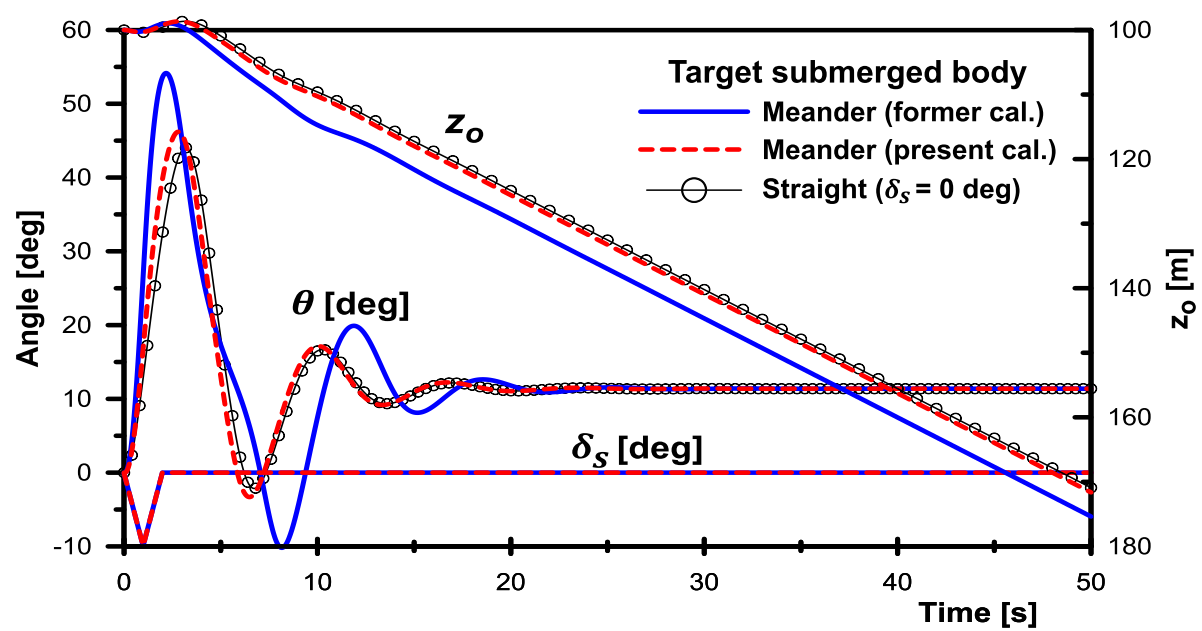

(b) Time series of pitch, stern plane and depth

Fig. 11 Meander maneuver simulation results of the target submerged body using formal and present control plane coefficients

\section{Conclusion}

Herein we conducted a case study to identify causes and take action when abnormal motions occurred during the numerical analysis. Based on numerical simulations, we developed a procedure for identifying parameters, the cause of abnormal motions. As a result of performing four steps of the procedure, the following conclusions are drawn.

1. First, stability analysis is done to check whether it is ensured and whether the system is capable of performing maneuvering simulation.

2. If the stability is ensured and the maneuvering simulation is feasible without applying controllers, parameters with a sensitive effect on dynamic characteristics are identified by carrying out sensitivity analysis which can reduce the number of coefficients that needs to be revalidated.

3. High-sensitivity maneuvering coefficients are compared with the ones obtained from captive model tests of other submerged bodies. If there are no captive model test results, they can be compared with coefficients calculated by using empirical formulae. 
4. Reanalysis should be done for coefficients that greatly exceed acceptable values differing from general cases.

5. By following the four steps above, we can identify problematic coefficients to help decision-making on mathematical models for submerged-body dynamics.

Limitations of the proposed method are as follows.

1. The proposed procedure may help reduce samples of maneuvering coefficients to be revalidated without suggesting a range of validity values for coefficients. Thus, if there is much difference in the shape of the operating mission between the target body and the pre-existing body, maneuvering coefficients should be compared with the ones calculated with empirical formulae.

2. Only the procedure for the correlation between maneuvering coefficients and abnormal motions is presented. Correlations between maneuvering coefficients and design parameters need to be analyzed separately.

\section{Acknowledgement}

The authors gratefully acknowledge the financial support of the Agency for Defense Development, the Republic of Korea under the project. This study is part of the results from the project of "Study on the model to track UUV's position of USV avoiding surface obstacles in waves".

\section{Conflicts of Interest}

The authors declare no conflict of interest.

\section{References}

[1] Y. K. Kim, K. H. Yun, S. Y. Kim, and D. J. Kim, "Captive model test of submerged body using CPMC," Journal of the Society of Naval Architects of Korea, vol. 49, no. 4, pp. 296-303, Aug. 2012.

[2] K. Y. Lee, "An analysis of required technologies for developing unmanned mine countermeasure system based on the unmanned underwater vehicle," Journal of the Korea Institute of Military Science and Technology, vol. 14, no. 4, pp. 579-589, Aug. 2011.

[3] J. Y. Park, N. Kim, K. P. Rhee, H. K. Yoon, C. Kim, C. Jung, K. Ahn, and S. Lee, "Study on coning motion test for submerged body," Journal of Ocean Engineering and Technology, vol. 29, no. 6, pp. 436-444, 2015.

[4] M. Jeon, H. K. Yoon, J. Hwang, and H. J. Cho, "Study on maneuvering characteristics of submerged body by changing its design parameters," Journal of Ocean Engineering and Technology, vol. 31, no. 2, pp. 155-163, 2017.

[5] Howaldtswerke-Deutsche Werft, "KSS- II course 14 manoeuvrability of submarine," Howaldtswerke-Deutsche Werft AG Report, Oct 15, 2001.

[6] E. A. Barros, A. Pascoal, and E. Sa, "AUV dynamics: modelling and parameter estimation using analytical, semi-empirical, and CFD method”, vol. 37, no. 10, pp. 369-376, Jul. 2004.

[7] T. T. Nguyen, H. K. Yoon, Y. Park, and C. Park, "Estimation of hydrodynamic derivatives of full-scale submarine using RANS solver," Journal of Ocean Engineering and Technology, vol. 32, no. 5, pp. 386-392, Oct. 2018.

[8] T. Gao, Y. Wang, Y. Pang, and J. Cao, "Hull shape optimization for autonomous underwater vehicles using CFD," Engineering Application of Computational Fluid Mechanics, vol. 10, no. 1, pp.599-607, Sep. 2016.

[9] Y. J. Sung and S. H. Park, "Prediction of Ship manoeuvring performance based on virtual captive model tests," Journal of the Society of Naval Architects of Korea, vol. 52, no. 5, pp. 407-417, Oct. 2015.

[10] H. Kim and H. Cho, "Numerical study on control derivatives of a high-speed underwater vehicle," Journal of Mechanical Science and Technology, vol. 25, no. 3, pp. 759, May. 2011.

[11] M. Z. Ernania, M. Bozorg, and S. Ebrahimi, "Identification of an autonomous underwater vehicle dynamic using extended kalman filter with ARMA noise model," International Journal of Robotics, vol. 4, no. 1, pp. 22-28, 2015.

[12] F. Azarsina, N. Bose, and M. S. Self, “An underwater vehicle maneuvering simulation; focus on turning maneuvers," The Journal of Ocean Technology, vol. 2, no. 1, pp. 54-73, 2007. 
[13] M. Jeon, H. K. Yoon, J. Hwang, and H. J. Cho, "Analysis of the dynamic characteristics for the change of design parameters of an underwater vehicle using sensitivity analysis," International Journal of Naval Architecture and Ocean Engineering, vol. 10, no. 4, pp. 508-519, Jul. 2018.

[14] A. J. Healey and D. Lienard, "Multivariable sliding-mode control for autonomous diving and steering of unmanned underwater vehicles,” IEEE Journal of Oceanic Engineering, vol. 18, no. 3, pp. 327-339, Jul. 1993.

[15] T. Prestero, "Development of a six-degree of freedom simulation model for the REMUS autonomous underwater vehicle," Proc. MTS/IEEE Oceans 2001, An Ocean Odyssey. Conference Proceedings(IEEE Cat. No. 01CH37295), Honolulu, HI, Nov. 2001, pp. 450-455.

[16] L. Lapierre and B. Jouvencel, "Robust nonlinear path-following control of an AUV," IEEE Journal of Oceanic Engineering, vol. 33, no. 2, pp. 89-102, Oct. 2008.

[17] J. Evans and M. Nahon, "Dynamics modeling and performance evaluation of an autonomous underwater vehicle," Ocean Engineering, vol. 31, no. 14-15, pp. 1835-1858, Oct. 2004.

[18] R. Yang, "Modeling and robust control approach for autonomous underwater vehicles," Ph.D. dissertation, Dept. Ocean Eng., Ocean Univ. of Chani., Cambridge, 2016.

[19] V.B.S. Ayyangar, P. Krishnankutty, M. Korulla, and P.K. Panigrahi, "Stability analysis of a positively buoyant underwater vehicle in vertical plane for a level flight at varying buoyancy, BG and speeds," Ocean Engineering, vol. 148, no. 15, pp. 331-348, Jan. 2018.

[20] D. J. Yeo, H. K. Yoon, Y. G. Kim, C. M. Lee, "Sensitivity analysis on the stability of a submarine concerning its design parameters," Journal of the Society of Naval Architects of Korea, vol. 43, no. 4, pp. 521-528, 2006.

[21] D. J. Yeo and K. P. Rhee, “A study on the sensitivity analysis of submersibles' manoeuvrability," Journal of the Society of Naval Architects of Korea, vol. 42, no. 5, pp. 458-465, 2005.

[22] D. J. Yeo and K. P. Rhee, "Design of sensitivity-maximizing input for submersibles' manoeuvring coefficients using genetic algorithm technique," Journal of the Society of Naval Architects of Korea, vol. 43, no. 2, pp. 156-163, 2006.

[23] D. Sen, "A study on sensitivity analysis of maneuverability performance on the hydrodynamic coefficients for submerged bodies,” Journal of Ship Research, vol. 44, no. 3, pp. 186-196, Nov. 2000.

[24] S. Thuné, "Simulation of Submarine Manoeuvring," Ms. Dissertation, Royal Institute of Technology, Sweden, 2015.

Copyright $\odot$ by the authors. Licensee TAETI, Taiwan. This article is an open access article distributed under the terms and conditions of the Creative Commons Attribution (CC BY-NC) license (https://creativecommons.org/licenses/by-nc/4.0/). 